\title{
Epidemiologia e Statistica per Tutti
}

\section{Una prima semplice applicazione: il Number Needed to Treat}

\author{
M. Nichelatti, M. Nordio, U. Maggiore, M. Postorino, A. Limido
}

a nome del Comitato Scientifico SIN-RIDT

\section{Ci vuole attenzione}

Abbiamo appena affrontato ciò che con molta sfacciataggine abbiamo chiamato "aspetto quantitativo della probabilità", e lo abbiamo identificato con la quantità di soggetti in cui si manifesta un certo evento, rispetto al totale dei soggetti, cioè con la frequenza dell'evento nei soggetti che stiamo osservando. Da questo, possiamo ricavare la probabilità (quella che abbiamo chiamato "intuitiva") che in un individuo appartenente a un campione estratto dalla popolazione si manifesti l'evento di nostro interesse, nello stesso modo in cui ricaviamo la probabilità di ottenere "testa" dopo il lancio di una moneta o " 6 " dopo il lancio di un dado. In pratica, quindi, se sappiamo che in una popolazione la malattia X (l'evento) si manifesta mediamente in 45 soggetti ogni mille, quando ci troviamo davanti a un soggetto di cui non conosciamo lo stato di salute rispetto a X, possiamo affermare che tale soggetto ha il $4.5 \%$ di probabilità di soffrire della malattia.

Tuttavia questa affermazione è un po' azzardata: infatti questa probabilità quantitativa è calcolata su una popolazione generale - o meglio - su un insieme di campioni estratti dalla popolazione, analizzati nella letteratura scientifica, e noi che esercitiamo l'attività in un ospedale non potremo mai conoscere direttamente la popolazione complessiva, ma solo un campione di essa, cioè i pazienti che accedono al nostro ospedale. E quale campione, poi? Un campione probabilmente selezionato in modo non casuale (almeno nella massima parte dei casi), almeno su base geografica, e quindi non sempre affidabile per trarre conclusioni definitive. Infatti, può darsi che il nostro ospedale sia un centro di riferimento per una data patologia, oppure che non lo sia affatto: nel primo caso, ci aspettiamo che la frequenza dei malati possa essere maggiore rispetto alla media, mentre nel secondo caso ci aspettiamo una frequenza probabilmente minore.

\section{Confrontare due frequenze}

Un problema di pratica clinica abbastanza sentito è il confronto delle frequenze con cui si ottiene un successo (un endpoint favorevole come una guarigione o una riduzione clinicamente significativa del dolore) o un fallimento (un decesso, una ricaduta) utilizzando un nuovo farmaco, rispetto al farmaco di riferimento per la patologia che stiamo studiando. Per evitare errori grossolani di valutazione, è bene effettuare il confronto traendo i risultati di interesse (le frequenze di efficacia) da trial clinici randomizzati, in modo che quanto viene asserito corrisponda ai necessari criteri di numerosità e di qualità e veridicità dei dati.

Per fare il confronto è necessario disporre di un evento chiaramente identificabile (prenderemo ad esempio la mortalità, utilizzando dei dati del tutto inventati) con un sì o con un no, senza ambiguità, e "riconosciuto" ufficialmente dalla letteratura scientifica. Ipotizziamo, a questo punto, di avere trovato, nei vari database clinici, che in una serie di protocolli randomizzati si è osservata, per una certa malattia X, la mortalità entro un mese dalla diagnosi in 630 casi su 1200 con il farmaco di riferimento (il farmaco di controllo), e in 450 casi su 1250 con il nuovo farmaco (il farmaco sperimentale).

Possiamo ottenere immediatamente la frequenza degli eventi (ER, dall'inglese Event Rate) nel gruppo di riferimento 
e nel gruppo sperimentale, che saranno rispettivamente uguali a

$$
\begin{aligned}
& \mathrm{CER}>\frac{630}{1200}>0.525>52.5 \% \\
& \mathrm{EER}>\frac{450}{1250}>0.36>36.0 \%
\end{aligned}
$$

troviamo quindi una CER (Control Event Rate) uguale a 0.525 (52.5\%) e una EER (Experimental Event Rate) pari a $0.36(36.0 \%)$.

\section{La riduzione del rischio}

Con il farmaco sperimentale, apparentemente, si muore di meno: possiamo immediatamente calcolare il rischio relativo di morte $(\mathrm{RR})$ che si ha quando si è trattati con il farmaco sperimentale anziché con lo standard, dato da

$$
\mathrm{RR}=\frac{\mathrm{EER}}{\mathrm{CER}}=\frac{0.36}{0.525} \approx 0.686=68.6 \% \text {, }
$$

pertanto, fatta 100 la mortalità dei soggetti in trattamento standard, quella dei soggetti in trattamento sperimentale diventa circa 68.8 .

D'altra parte, anche soggetti sottoposti al trattamento sperimentale muoiono, e quindi non sarebbe corretto attribuire al trattamento standard tutta la mortalità. Si fa quindi ricorso al calcolo della riduzione del rischio ottenuta con il cambio del trattamento da standard a sperimentale: possiamo definire una riduzione del rischio relativa (RRR), ed una assoluta (ARR); la prima è calcolata come

$$
\mathrm{RRR}=\frac{\mathrm{CER}-\mathrm{EER}}{\mathrm{CER}}=\frac{\mathrm{AR}}{\mathrm{CER}}=\frac{0.165}{0.525} \approx 0.314=31.4 \%,
$$

mentre la seconda è data da

$$
\mathrm{ARR}=\mathrm{CER}-\mathrm{EER}=0.525-0.36=0.165=16.5 \% \text {. }
$$

L'ARR è una variabile di grande importanza, perché consente di calcolare il Number Needed to Treat (NNT, talvolta chiamato anche NNEE, Number Needed for Extra Effect), ovvero il numero di pazienti da trattare con il farmaco sperimentale per evitare un evento. L'NNT è semplicemente il reciproco dell'ARR, per cui

$$
\mathrm{NNT}=\frac{1}{\mathrm{ARR}}
$$

e quindi, nel nostro caso,

$$
\mathrm{NNT}=\frac{1}{\mathrm{ARR}}=\frac{1}{0.165} \approx 6.06,
$$

che, arrotondato per ovvi motivi di prudenza all'intero più basso, dà NNT $=6$. 


\section{Come si interpreta l'NNT}

Abbiamo appena visto che nel nostro caso, l'uso di un nuovo farmaco sperimentale contro la malattia X riduce la mortalità, con un NNT pari a 6: questo implica che ogni 6 pazienti trattati con il farmaco sperimentale si risparmia un decesso, oppure - il che è lo stesso, anche se cambia il punto di vista - che occorre trattare 6 pazienti con il farmaco nuovo per avere un decesso in meno. Questa è l'interpretazione statistico-clinica, che è una cosa, ma occorre considerare anche una seconda chiave interpretativa, diciamo epidemiologico-politica, che è un'altra cosa.

Ovviamente il farmaco perfetto, ed anche la decisione perfetta (ad esempio, vaccinare o non vaccinare una popolazione per evitare una malattia) hanno $\mathrm{NNT}=1$, cioè basta trattare un soggetto malato (a rischio, in caso di una vaccinazione) per evitare un evento singolo, cioè, in ogni soggetto trattato l'evento non si manifesta; invece, quanto più elevato è il valore dell'NNT, tanto maggiore sarà il numero di soggetti da trattare per evitare che si verifichi un singolo evento che invece si verificherebbe comunque con il trattamento standard. Potremo quindi avere, ad esempio, un valore come $\mathrm{NNT}=1000$, che ci dice che occorre trattare 1000 soggetti a rischio con il nuovo farmaco per ridurre di una unità il numero di eventi che conteremmo se intervenissimo nel modo tradizionale, ed occorre stare molto attenti davanti a questa affermazione, perché la riduzione di una unità è completamente indipendente dal numero di eventi osservati con il trattamento standard. Quindi, trattando con il nuovo farmaco caratterizzato da NNT $=1000$, avremo 999 eventi se l'evento si manifestava in tutti i soggetti trattati con il farmaco standard, e 0 eventi se l'evento si manifestava solo in uno dei soggetti trattati con lo standard.

Ripetiamo il concetto, perché è importante: il numero di eventi che si contano utilizzando la terapia standard è irrilevante: la cosa importante è che, cambiando la terapia da standard a sperimentale, il numero di eventi sarà uno in meno. Possiamo verificare la nostra affermazione in modo molto semplice: infatti, dire NNT = 1000 significa

$$
\mathrm{NNT}=\frac{1}{\mathrm{ARR}}=\frac{1}{\mathrm{CER}-\mathrm{EER}}=1000,
$$

e quindi che CER-EER $=0.001=0.1 \%$. Questo vale quando $\mathrm{CER}=0.234$ e EER $=0.233$, o quando $\mathrm{CER}=0.651$ e EER $=0.650$, e così via: in pratica, è sufficiente che la differenza tra CER e EER sia 0.001, e NNT sarà comunque uguale a 1000, indipendentemente dal valore di CER.

A questo punto, devono intervenire delle riflessioni anche di carattere politico (di politica sanitaria, s'intende): qual è la gravità della patologia interessata, e qual è l'allarme sociale che questa innesca? È evidente che una malattia che colpisce i bambini, magari ad elevata mortalità desta un allarme sociale molto maggiore di una malattia altrettanto mortale che colpisce gli ottantenni; ed è altrettanto evidente che l'allarme sociale è anche una funzione inversa della distanza spaziale che separa un paese dal luogo in cui la malattia si manifesta.

Ma non è tutto: qual è il costo del trattamento con i due metodi? Cioè, vale la pena spendere per il nuovo trattamento? Molto probabilmente, la risposta sarà tanto più vicina al sì quanto minore il valore del NNT ottenuto.

Finora abbiamo assunto di avere CER > EER e che l'evento di nostro interesse sia "negativo" (un decesso), ma nulla cambierebbe se avessimo EER > CER, oppure se l'evento fosse positivo (una guarigione): è sufficiente interpretare i risultati. In generale, comunque, quando $\mathrm{EER}>\mathrm{CER}$, si usa la differenza $\mathrm{ABI}=\mathrm{EER}-\mathrm{CER}$, dove $\mathrm{ABI}$ sta per Absolute Benefit Increase; ovviamente, in questo caso, avremo NNT = 1/ABI.

\section{Domande}

Calcolare l'NNT nelle seguenti situazioni:

- Decessi con trattamento tradizionale: 146 casi su 7020; decessi con il nuovo farmaco: 67 casi su 4110.

- Guarigioni con trattamento tradizionale: 278 casi su 361; guarigioni con il nuovo farmaco: 221 casi su 300. 


\section{Risposte alle domande nel numero precedente}

- Basta moltiplicare 87 mila per 1.43 per ottenere un capitale attuale pari a 124410.

- Le azioni della società $X$, al secondo giorno valgono $(500 \times 1.1) \times 0.9=495$, mentre quelle della società $Y$, sempre al secondo giorno valgono $(500 \times 0.9) \times 1.1=495$, per cui il povero signor Pasquale, oltre a non saper fare $i$ conti, è anche decisamente sfortunato nei suoi investimenti.

- È più efficace il farmaco B (vedere la Tabella), malgrado le apparenze, e checché ne pensino i due Direttori (anche loro, come il signor Pasquale, non sanno fare i conti)

\begin{tabular}{|c|c|c|c|}
\hline & \multicolumn{2}{|c|}{ FARMACO A } & \multirow{2}{*}{ \% efficacia } \\
\hline & EFFICACE & NON EFFICACE & \\
\hline \multirow[t]{3}{*}{ Medicina 1+2 } & 53 & 47 & $53 \%$ \\
\hline & \multicolumn{2}{|c|}{ FARMACO B } & of sfingui \\
\hline & EFFICACE & NON EFFICACE & to enicdacid \\
\hline Medicina 1+2 & 67 & 33 & $67 \%$ \\
\hline
\end{tabular}

- La probabilità di fare " 13 ” $\grave{e}\left(\frac{1}{3}\right)^{13}=\frac{1}{1594323} \approx 6.27225 \times 10^{-7}$

- La probabilità di fare " $O$ ” $\grave{e}\left(\frac{2}{3}\right)^{13}=\frac{8192}{1594323} \approx 5.13823 \times 10^{-3}$;

(fare "O" è più facile perché la probabilità di indovinare il risultato è $1 / 3$, mentre quella di non indovinarlo è doppia).

- Lanciando due dadi, ci sono 5 combinazioni che fanno ottenere 8 (2+6, 3+5, 4+4, 5+3, 6+2), 4 combinazioni che fanno ottenere $9(3+6,4+5,5+4,6+3)$, e 3, 2 e 1 combinazioni che fanno ottenere rispettivamente 10, 11 e 12; le combinazioni di nostro interesse sono $5+4+3+2+1=15$, per cui la probabilità di ottenere almeno $8 \grave{e} 15 / 36 \approx 0.4167=41.67 \%$.

- I possibili risultati dati da un numero pari sono 2 (1 combinazione), 4 (3 combinazioni), 6 (5 combinazioni), 8 (5 combinazioni), 10 (3 combinazioni) e 12 (1 combinazione), per cui le combinazioni favorevoli sono 18, e quindi la probabilità di un risultato pari è 18/36=0.5=50.0\%.

Le risposte alle domande presenti nell'articolo saranno pubblicate sul prossimo numero del Giornale di Tecniche Nefrologiche \& Dialitiche Vol. 25, No. 1 